\title{
CORRECTED ACOUSTIC POYNTING VECTOR AND ITS APPLICATION IN REVERSE-TIME MIGRATION
}

\author{
Lucas Ferreira Conz Eugenio id and Leandro Di Bartolo (D)
}

\begin{abstract}
Reverse-time migration (RTM) is the most important seismic imaging method for complex subsurface geology. However, the simple use of the traditionally applied cross-correlation imaging condition leads to a strong lowfrequency noise in the final images. Many studies have proposed solutions to this problem, either by applying filters in the image domain, such as the Laplacian filter, or by developing new imaging conditions. One example is the imaging condition that correlates only the wavefield components with opposite propagation directions in forward and backward extrapolation. In this paper, it was applied the latter technique using the acoustic Poynting vector to determine the propagation directions. The wavefield extrapolation uses a staggered-grid finite difference scheme. To guarantee that the displacement velocities are considered at the correct grid position, it was applied a linear interpolation in the Poynting vector. This procedure can improve the wavefield decomposition and the final migration results.
\end{abstract}

Keywords: Marmousi model, denoising, noise suppression, acoustic wavefield

RESUMO. A migração reversa no tempo é o método de imageamento sísmico mais importante para geologias de subsuperfície complexas. No entanto, o uso da tradicionalmente aplicada condição de imagem de correlação cruzada introduz fortes ruídos de baixa frequência nas imagens finais. Muitos estudos propuseram soluções para este problema, seja aplicando filtros no domínio da imagem, como o filtro Laplaciano, ou desenvolvendo novas condições de imagem. Um exemplo é a condição de imagem que correlaciona somente as componentes do campo de ondas cujas direções de propagação sejam opostas na extrapolação direta e reversa. Nesse artigo, foi aplicada esta última técnica utilizando o vetor de Poynting para determinar as direções de propagação. A extrapolação de campos de ondas utiliza um esquema de diferenças finitas de malhas intercaladas. Para garantir que os campos de deslocamento de velocidades sejam considerados na correta posição da malha, uma interpolação linear foi aplicada no vetor de Poynting. Esse procedimento pode melhorar a decomposição do campo de ondas e os resultados finais de migração.

Palavras-chave: Modelo Marmousi, supressão de ruído, campo de ondas acústico 


\section{INTRODUCTION}

Reverse-time migration (RTM) is a migration technique based on the full wavefield extrapolation from source and receiver sides, commonly applied in the pre-stack dataset. It was first introduced in the 1980s by Baysal et al. (1983), McMechan (1983), Whitmore (1983) and Loewenthal \& Mufti (1983) when its application to real datasets was not possible due to processing limitations in time and memory.

However, after the large increase in processing and storage provided by technology advances, mainly after the 2000s, RTM algorithms have been in evidence in seismic imaging workflows due to their ability to generate good results in complex environments.

RTM algorithms basically consist of two main steps: (1) forward wavefield extrapolation from source position through the subsurface model using a modelled source and (2) backward wavefield extrapolation from receiver positions using the inverse of the seismograms as source. During this second step, an imaging condition is applied to construct the subsurface image. The imaging condition is not unique and many imaging conditions with different properties, advantages and disadvantages can be applied.

As the RTM final image is very sensitive to the chosen imaging condition, different conditions were proposed to obtain a better image of the subsurface. The most conventional imaging condition used is known as cross-correlation, which correlates the source and receiver full wavefields through the entire wave path of the first and second RTM steps. However, this imaging condition generates the wellknown low-frequency high-amplitude back scattered noise in the final images.

Once this problem was detected, many authors have proposed different imaging condition techniques to deal with these artifacts as: Fletcher et al. (2005) that proposed a damp directional factor, Guitton et al. (2007) which proposed a least squares solution to minimize those noises, Zhang \& Sun (2009) utilized the application of the Laplacian filter to remove the low-frequency artifacts and Yoon \& Marfurt (2006) introduced the concept of Poynting vector to improve the conventional cross-correlation imaging condition.

Recently, new imaging conditions based on the full wavefield decomposition are being widely studied and proposed. There are many ways to reach this wavefield separation in its up and down-going components. It can be achieved by 1) the application of the Fast Fourier Transform (FFT) and the splitting of the components in the F-K domain (Suprajitno \& Greenhalgh, 1985; Hu \& McMechan, 1987); 2) by using the Poynting vector concept which indicates the flux of energy per unit of area (Liu et al., 2011; Chen and He, 2014; Ren et al., 2015) and, hence, the direction of propagation of the seismic wave and 3) through the application of the analytical wavefield which is based on two propagations, one using the conventional source and other using the Hilbert transformed source (Revelo \& Pestana, 2019).

Each one of these techniques has its advantages and disadvantages but all of them have the memory increase disadvantage in comparison with the conventional cross-correlation imaging condition, once they decompose the full wavefield in two wavefields (up and down-going) or even in four wavefields when the left and right-going components are also considered.

However, in terms of time of processing, the use of FFT is more expensive than the use of the Poynting vector method, as the second does not need to perform a Fourier transform at each time step. The recent analytical wavefield is the most efficient condition, as it does not need to execute data transformations through the wavefield propagations, only on the seismic source and, therefore, it is being considered the best option on algorithms that use the RTM imaging condition based on wavefield decompositions (Revelo \& Pestana, 2019).

Many authors as Liu et al. (2011), Chen \& He (2014) and Ren et al. (2015) have proposed new imaging conditions based on the acoustic Poynting vector wavefield decomposition. Shi et al. (2019) extrapolated the use of this method to the elastic formulation, proposing its use in 2D elastic RTM. 
Also, Chen \& McMechan (2018) identified a problem with the dynamic of the acoustic Poynting vector proposed by Yoon \& Marfurt (2006) and have developed a dynamically corrected acoustic Poynting vector. Costa et al., (2009) have proposed an obliquity correction to the Poynting vector imaging condition to improve the RTM image by reducing the backscattering noise.

The Poynting vector wavefield separation technique is based on the use of a signal criteria to split the wavefield components. This signal is evaluated on the product between the Poynting vector and the particle velocity vector. In general, the particle velocity vector is obtained using a staggered grid which calculates the velocities in shifted positions in comparison with the pressure acoustic field. If this particularity is not considered, the decomposition becomes incomplete, as remaining amplitudes of the down-going wavefield are present in the up-going wavefield and vice versa. Also, if the separation is made without using a proper taper in the intersection points of the two wavefields, the generated image will contain artifacts in the form of chopped marks right above the geological interfaces.

In this paper, we present a study about the Poynting vector decomposition when using an ordinary staggered grid scheme to calculate the acoustic wavefields and its particularities during the separation of the full acoustic wavefield. An interpolation of the Poynting vector is proposed to solve the problem of the staggered grid calculation and, therefore, completely achieve the separation of the up and down-going components without residuals.

\section{METHODOLOGY}

The methodology used in this paper is described in this section. The first part is dedicated to describe the acoustic formulation used in this paper, as well as the finite-difference method used to solve numerically all equations of this formulation. The second part is dedicated to the explanation on how the acoustic Poynting vector was obtained and to the introduction of the wavefield separation criteria utilized in this paper.

\section{Acoustic Wave Equation}

The acoustic wave-equation used in this paper for an isotropic medium is

$$
\begin{gathered}
\frac{\partial v_{x}}{\partial t}=-\frac{1}{\rho}\left(\frac{\partial p}{\partial x}\right), \\
\frac{\partial v_{z}}{\partial t}=-\frac{1}{\rho}\left(\frac{\partial p}{\partial z}\right), \\
\frac{\partial p}{\partial t}=-\rho v\left(\frac{\partial v_{x}}{\partial x}+\frac{\partial v_{z}}{\partial z}\right) ;
\end{gathered}
$$

where $v_{x}$ and $v_{z}$ are the particle velocities in the $x$ and $z$ directions, respectively; $t$ is time; $p$ is the pressure field; $v$ and $\rho$ are respectively the compressional velocity and the density of the medium. The density adopted in this paper is constant and has the water value of $1,000.0 \mathrm{~kg} / \mathrm{m}^{3}$.

\section{Finite-Difference Method}

The finite-difference method will be used to numerically solve the bi-dimensional acoustic wave equations used in this paper. A second order in time and fourth order in space ordinary staggered grid (OSG) was used to discretize the equations.

The OSG grid was chosen once it provides all the ingredients used to generate the Poynting vector in the wavefield separation step. The central finite-difference operators were obtained by Taylor series expansion around each node in the staggered grid and the continuous equations become as follows:

$$
\begin{aligned}
& U_{i+1 / 2, j}^{k+1 / 2}= U_{i+1 / 2, j}^{k-1 / 2}-\frac{\Delta t}{24 h \rho_{i, j}}\left(P_{i-1, j}^{k}-\right. \\
&\left.27 P_{i, j}^{k}+27 P_{i+1, j}^{k}-P_{i+2, j}^{k}\right), \\
& V_{i, j+1 / 2}^{k+1 / 2}= V_{i, j+1 / 2}^{k-1 / 2}-\frac{\Delta t}{24 h \rho_{i, j}}\left(P_{i, j-1}^{k}-\right. \\
&\left.27 P_{i, j}^{k}+27 P_{i, j+1}^{k}-P_{i, j+2}^{k}\right),
\end{aligned}
$$




$$
\begin{aligned}
P_{i, j}^{k+1}= & P_{i, j}^{k}-\frac{\Delta t \rho_{i, j}}{24 h}\left[\left(U_{i-3 / 2, j}^{k+1 / 2}-\right.\right. \\
& 27 U_{i+1 / 2, j}^{k+1 / 2}+27 U_{i+1 / 2, j}^{k+1 / 2}- \\
& \left.U_{i+3 / 2, j}^{k+1 / 2}\right)+\left(V_{i, j-3 / 2}^{k+1 / 2}-\right. \\
& 27 V_{i, j-1 / 2}^{k+1 / 2}+27 V_{i, j+1 / 2}^{k+1 / 2}- \\
& \left.\left.V_{i, j+3 / 2}^{k+1 / 2}\right)\right]-\Delta t \rho_{i, j} c_{i, j}^{2} S_{i, j}
\end{aligned}
$$

where $h$ is the spacing of the mesh in both directions $(x, z) ; \rho_{i, j}$ and $c_{i, j}$ are the density and velocity of the mediums; $\Delta t$ is the time interval between two consecutive iterations during the wave propagation; $S_{i, j}$ is the source term; and $P, U, V$ are the discrete field of pressures and the particle velocities components in $x$ and $z$, respectively. The source used is the well known first time derivative of the Gaussian function, also called Ricker.

\section{Acoustic Poynting Vector}

The acoustic Poynting vector was first introduced by Yoon \& Marfurt (2006) and is an analogy to the original concept from electromagnetic waves. It indicates the flux of energy per unit of area and hence the direction of the propagation of the seismic wave.

The acoustic Poynting vector can be calculated by the product between the pressure field and the particle velocities as follows:

$$
\boldsymbol{S}(t, \vec{x})=-p(t, \vec{x}) \boldsymbol{v}(t, \vec{x})
$$

where $S$ is the Poynting vector; $p$ is the acoustic pressure field; and $v$ is the particle velocity vector, which in 2D has the vertical $\left(v_{z}\right)$ and the horizontal $\left(v_{x}\right)$ components. Therefore, one can easily obtain the horizontal $\left(S_{x}\right)$ and vertical $\left(S_{z}\right)$ components of the Poynting vector.

\section{Wavefield Separation}

It is possible to use both components of the Poynting vector to obtain the four components of the wavefield. Using the horizontal component, one can obtain the right and left-going wavefields; instead, using the vertical component, one can obtain the up and down-going wavefields.
Following Chen \& He (2014) and Ren et al. (2015), the criteria utilized to separate the full wavefield by using the acoustic wavefield and obtain the vertical components is defined below:

$$
\begin{aligned}
& p_{u}(t, \vec{x})=\left\{\begin{array}{c}
p(t, \vec{x}), \text { if } S_{z} \geq 0 \\
0, \text { if } S_{z}<0 .
\end{array}\right. \\
& p_{d}(t, \vec{x})=\left\{\begin{array}{c}
0, \text { if } S_{z} \geq 0 \\
p(t, \vec{x}), \text { if } S_{z}<0 .
\end{array}\right.
\end{aligned}
$$

In Eq. (8) and Eq. (9), $p$ is the full wavefield; $p_{u}$ and $p_{d}$ are respectively the up and down-going wavefields; and $S_{Z}$ is the vertical component of the acoustic Poynting vector.

\section{RESULTS AND DISCUSSION}

This section is dedicated to present the results obtained in this paper and to provide a proper discussion on them.

\section{Poynting vector Interpolation}

The OSG formulation to calculate the acoustic wavefield propagation naturally provides the particle velocity components at each time step, which is the main reason why this formulation was chosen.

However, if one looks at the OSG mesh in Figure 1 from Di Bartolo et al. (2012), the particle velocity components $\left(v_{x}, v_{z}\right)$ are calculated in dislocated positions (half sample) in comparison with the pressure field $(p)$.

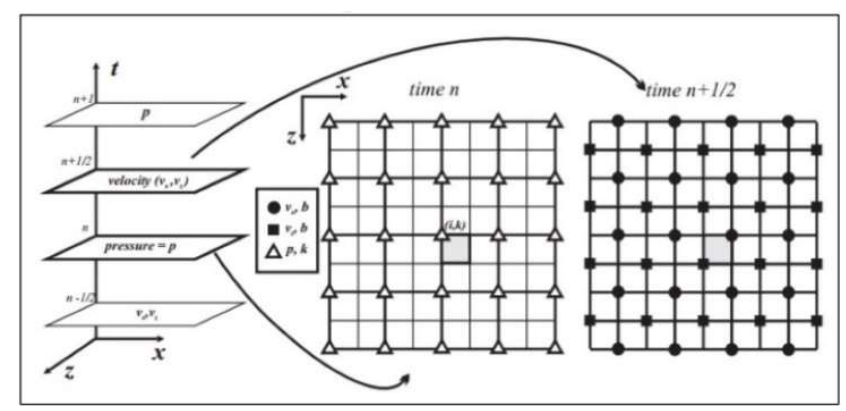

Figure 1 - Mesh grid scheme from Di Bartolo et al. (2012). 
Therefore, the product of the Eq. (7) provides a shifted Poynting vector - compared to the acoustic wavefield - when using this kind of mesh. If this issue is not properly handled when calculating the Poynting vector, the wavefield separation will not be completely achieved.

This statement is supported by the results in Figure 2. In this figure, one can see an experiment provided by a separation performed in a homogeneous medium with $\mathrm{P}$-wave velocity of $1,500.0 \mathrm{~m} / \mathrm{s}$ where the source is located at the center of this medium.

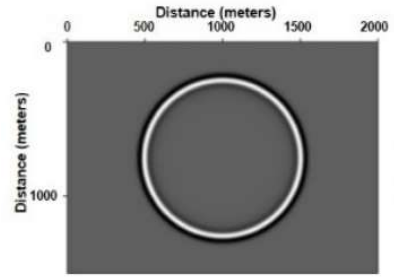

(a)

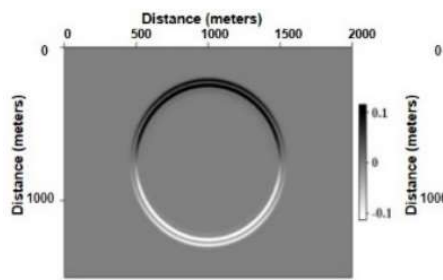

(c)

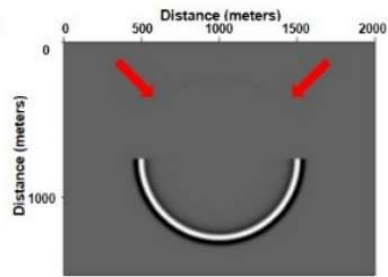

(b)

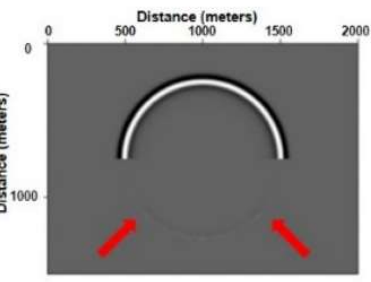

(d)
Figure 2 - Different frames in the same time step during the wavefield propagation illustrating the decomposition. In (a) the full wavefield, (b) down-going wavefield, (c) vertical Poynting vector and (d) up-going wavefield.

The four frames are representing snapshots of the full wavefield, the respective Poynting vector and the up and down-going components generated. All of them are referent to the same time step.

The conclusion is that one can easily see the residuals, pointed by the red arrows, present in the decomposed wavefields, and it may become worse when the propagation occurs in complex mediums where RTM is used. However, the differences on the imaging results by using the interpolated wavefield are not dramatic in comparison with the non-interpolated version.
In order to achieve a full wavefield separation, it is proposed an interpolation in the Poynting vector wavefield in both directions to shift back the calculated Poynting vector to the same position in relation to the pressure field before the application of the separation criteria.

The interpolation performed was a simple linear interpolation to obtain the middle point values as the shift in the mesh is exactly a half sample. Figure 3 shows the same experiment from Figure 2, but now with the proposed interpolation before the application of Eqs. (8) and (9).

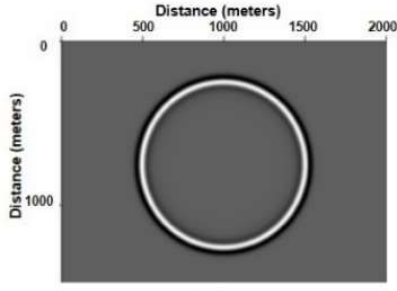

(a)

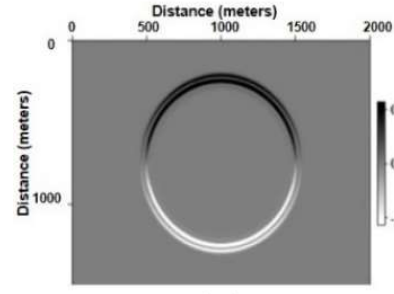

(c)

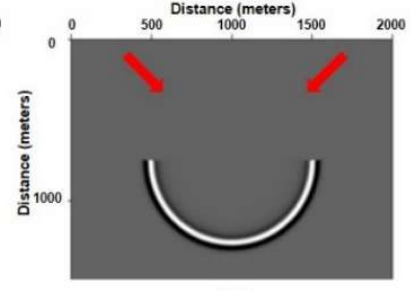

(b)

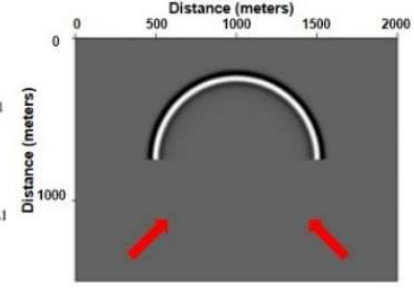

(d)
Figure 3 - Same model and frames of Figure 2 but this time using the proposed interpolation prior to the separation criteria. In (a) the full wavefield, (b) down-going wavefield, (c) vertical Poynting vector and (d) up-going wavefield.

\section{Marmousi Model}

The 2D Marmousi model (Versteeg \& Grau, 1990) was the first chosen to demonstrate the efficiency of the developed RTM algorithm (Fig. 4).

The dataset was first migrated using the conventional RTM imaging condition of crosscorrelation. The next step was to migrate using the developed RTM algorithm with the imaging condition based on the wavefield decomposition applying the interpolation of the Poynting vector (Fig. 5). 


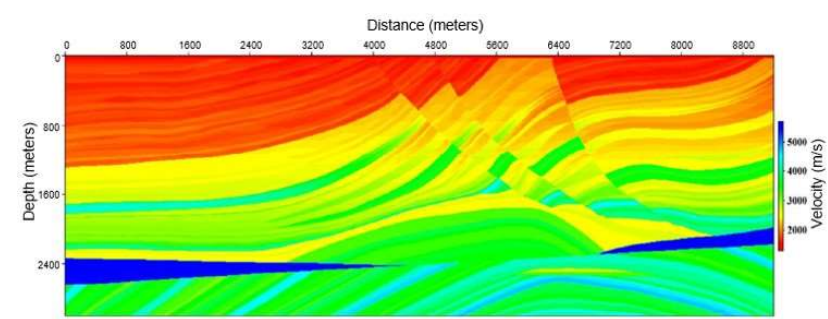

Figure 4 - 2D Marmousi velocity model.

The image produced by the conventional imaging condition contains the well-known lowfrequency artifacts while the image produced by the proposed algorithm illustrates the efficiency on the attenuation of these backscattering noises.

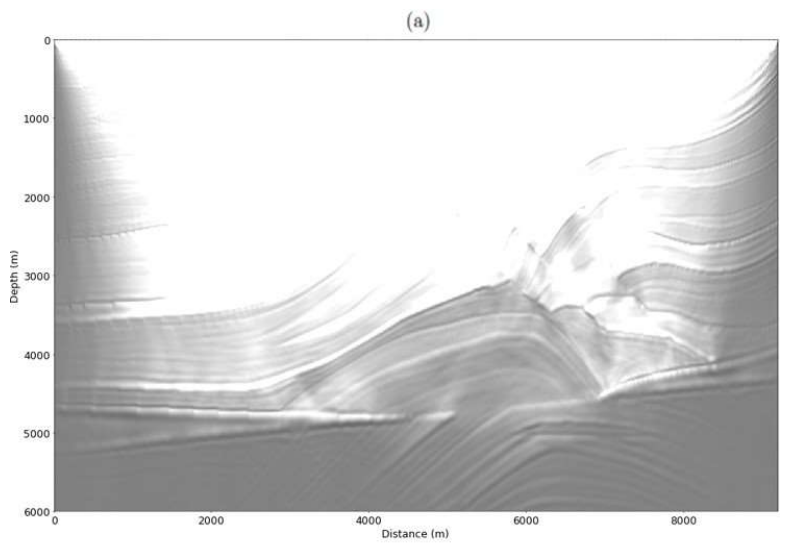

(b)

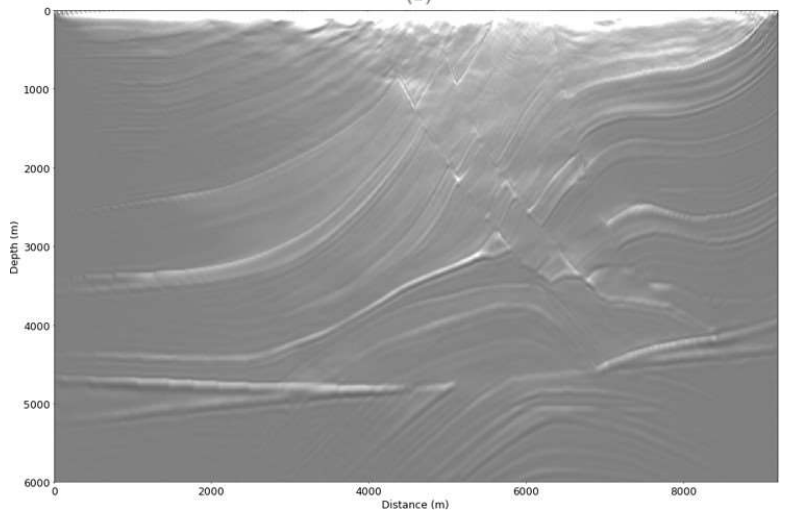

Figure 5 - Final Marmousi RTM images obtained using: (a) the conventional cross-correlation imaging condition; (b) the imaging condition based on wavefield decomposition (only vertical component).

Another important point to highlight is that there is no need of velocity model smoothing when using the RTM imaging condition based on the wavefields decomposition. This methodology is commonly used to attenuate the backscattering in the conventional cross-correlation.

However, it is important to mention that there is still room for improvement in this image, especially because in this paper it was only applied the vertical decomposition of the full wavefield. Therefore, the remnant low-frequency noise and the image quality could be further improved using the vertical component.

\section{Sigsbee Model}

The Sigsbee model, in its 2nd version, was also used to validate the RTM algorithm and results (Fig. 6). This model was created by the SMAART consortium, composed by BHP Petroleum, BP, Chevron and Texaco with the goal to test migration algorithms developed to improve the imaging quality in highly complex geological structures, as the ones associated with salt bodies.

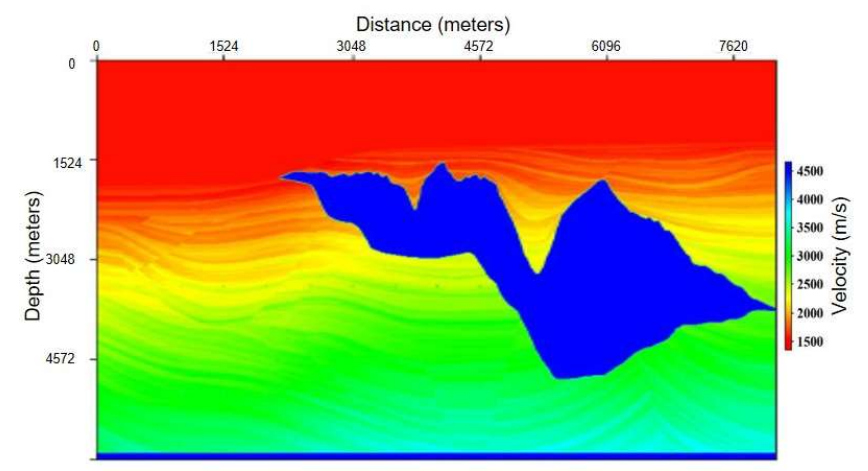

Figure 6 - Sigsbee 2a velocity model.

The results obtained using the conventional cross-correlation imaging condition and the one based on the wavefield decomposition can be seen in Figure 7. In this case, the backscattering noise is even stronger because of the geometry of the salt body.

One can see that the proposed algorithm successfully attenuates the low-frequency artifact and, therefore, the image quality becomes increased. 
(a)

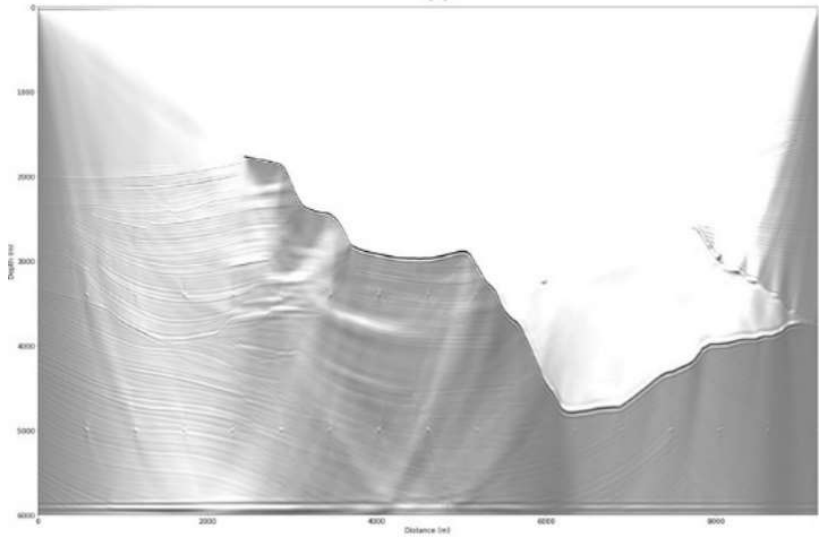

(b)

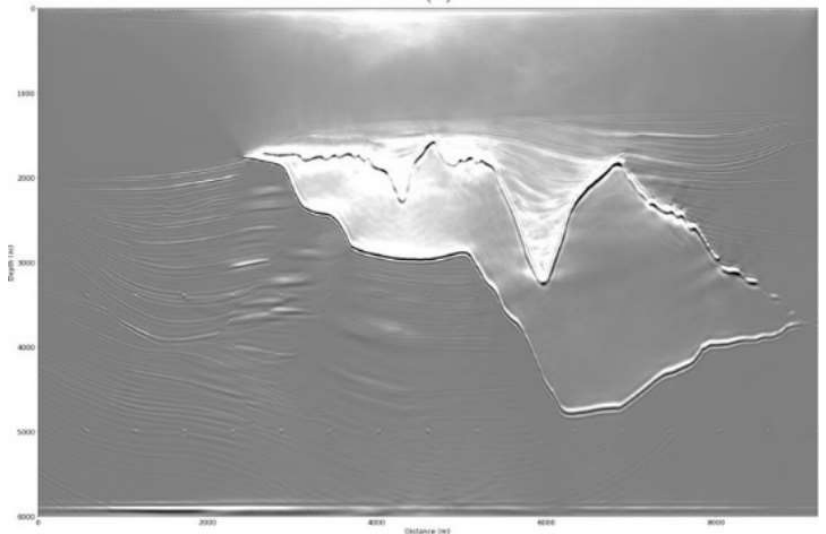

Figure 7 - Final Sigsbee 2a RTM images obtained using: (a) the conventional cross-correlation imaging condition; (b) the imaging condition based on wavefield decomposition (only vertical component).

\section{CONCLUSION}

It was proposed in this paper the interpolation of the Poynting vector to correct its actual position with respect to the pressure field. The results showed that this proposition provided a more accurate wavefield separation illustrated by the up and down-going components.

The images obtained with the imaging condition based on the wavefield decomposition (vertical components only) using the corrected Poynting vector provided better results than the images generated with the application of the conventional cross-correlation technique by reducing the low-frequency noise, also referred as backscattering artifacts.

\section{REFERENCES}

BAYSAL E, KOSLOFF DD \& SHERWOOD JWC. 1983. Reverse time migration. Geophysics, 48: 1514-1524.

CHEN T \& HE B. 2014. A normalized wavefield separation cross-correlation imaging condition for reverse time migration based on Poynting vector. Journal of Applied Geophysics, 11: 158-166.

CHEN T \& McMECHAN A. 2018. The dynamically correct Poynting vector formulation for acoustic media with application in calculating multidirectional propagation vectors to produce angle gathers from reverse time migration. Geophysics, 83: S365-S374.

COSTA JC, SILVA NETO FA, ALCÂNTARA MRIAN M, SCHLEICHER J \& NOVAIS A. 2009. Obliquity correction imaging condition for reverse time migration. Geophysics, 74: S57-S66.

DI BARTOLO L, DORS C \& MANSUR WJ. 2012. A new family of finite-difference schemes to solve the heterogeneous acoustic wave equation. Geophysics, 77: T187-T199.

FLETCHER RR, FOWLER P \& KITCHENSIDE P. 2005. Suppressing artifacts in prestack reversetime migration. SEG Technical Program Expanded Abstracts, p. 2049-2051.

GUITTON A, KAELIN B \& BIONDI B. 2007. Leastsquares attenuation of reverse time migration artifacts. Geophysics, 72: S19-S23.

HU L \& McMECHAN GA. 1987. Wave-field transformations of vertical seismic profiles. Geophysics, 52: 307-321.

LIU F, ZHANG G \& MORTON SA. 2011. An effective imaging condition for reverse-time migration using wavefield decomposition. Geophysics, 76: S29-S39.

LOEWENTHAL D \& MUFTI I. 1983. Reversed time migration in spatial frequency domain. Geophysics, 48: 627-635.

McMECHAN GA. 1983. Migration by extrapolation of time-dependent boundary values. Geophysical Prospecting, 31: 413-420. 
REN C, SONG G \& TIAN X. 2015, The use of Poynting vector in wave-field decomposition imaging condition for reverse-time migration. Journal of Applied Geophysics, 112: 14-19.

REVELO DE \& PESTANA RC. 2019, Up/down acoustic wavefield decomposition using a single propagation and its application in reverse time migration. Geophysics, 84: S341-S353.

SHI Y, ZHANG W, WANG Y. 2019, Seismic elastic RTM with vector-wavefield decomposition. Journal of Geophysics and Engineering, 16: 509-524.

SUPRAJITNO M \& GREENHALGH A. 1985, Separation of upgoing and downgoing waves in vertical seismic profiling by contour-slice filtering. Geophysics, 50: 892-1047.

L.F.C.E.: Responsible for the execution and development of the paper as a consequence of the results obtained throughout the Master program of Geophysics at Observatório Nacional. L.D.B.: Responsible for orientation, suggestions and revisions of the submitted paper.

Received on February 21, 2020 / Accepted on December 28, 2021

Recebido em 21 de fevereiro de 2020 / Aceito em 28 de dezembro de 2021
VERSTEEG R \& GRAU G. 1990, Practical aspects of seismic data inversion, the Marmousi experience. In: Proceedings of the $52^{\text {nd }}$ EAGE Meeting, Copenhagen, Denmark.

WHITMORE ND. 1983, Iterative depth migration by backward time propagation. SEG Technical Program Expanded Abstracts, 382-385.

YOON K \& MARFURT K. 2006, Reverse-time migration using the Poynting vector. Exploration Geophysics, 37: 102-107.

ZHANG Y \& SUN J. 2009, Practical issues in reverse-time migration. True amplitude gathers, noise removal and harmonic-source encoding. First Break, 27: 53-59. 